\title{
Design and Implementation of DAG-Based Co-scheduling of RPC in the Grid
}

\author{
JiHyun Choi ${ }^{1}$, DongWoo Lee ${ }^{2}$, R.S. Ramakrishna ${ }^{3}$, \\ Michael Thomas ${ }^{4}$, and Harvey Newman ${ }^{5}$ \\ ${ }^{1,2,3}$ Department of Information and Communication, \\ Gwangju Institute of Science and Technology, Republic of Korea \\ \{jhchoi80, leepro, rsr\}@gist.ac.kr \\ 1,4,5 California Institute of Technology, Pasadena, CA91125, USA \\ \{jchoi, thomas, newman\}@hep.caltech.edu
}

\begin{abstract}
Effective scheduling in the Grid consisting of heterogeneous and distributed resources is imperative in order to counter unacceptably large overheads of the Grid. We proposed the grid middleware (pyBubble) supporting the DAG based co-scheduling for improving the performance of the RPC mechanism. DAG based co-scheduling reduces redundant transmission of input and output data from execution of the sequence of related client requests, thereby decongesting the network. We demonstrate the efficiency of DAG based co-scheduled RPC in experiments compared with the overhead of the traditional RPC mechanism.
\end{abstract}

\section{Introduction}

The Grid is the Internet-connected computing and data management infrastructure. Computing and data resources are geographically dispersed in different administrative domains with different policies for security and resource usage. The computing resources are highly heterogeneous, ranging from single PCs and workstations, cluster of workstations, to large supercomputers [5]. With the technology of the Grid we can construct large-scale and scientific applications over these distributed and heterogeneous resources. There are many critical issues that need to be efficiently resolved to support the ever-increasing number of applications that can benefit from the Grid Computing infrastructure.

GridRPC 8 is a programming model based on client-server remote procedure call(RPC), with features added to allow easy programming and maintenance of code for scientific applications on the Grid. Application programmers write parallelized client programs using simple and intuitive GridRPC APIs that hide most of the complexities involving Grid programming. As a result, programmers lacking experience in parallel programming, let alone the Grid, can still construct Grid Applications effortlessly 3 . Most applications in Grid Computing generally have large input data sets and intricate data dependency. Moreover, data transfer in large distributed systems can add an unacceptable amount of overhead. The 
goal of this research is to devise simple and effective strategies for dealing with these issues.

\section{Related Works}

Grid programming involves the Interface and the Run-time system. WS-Resource Framework defines Web service convention to enable the discovery of, introspection on, and interaction with stateful resources in standard and interoperability ways. Many enhanced interfaces for grid computing such as Grid MPI and Grid RPC have been developed. With regard to Message Passing Interface(MPI), several systems, notably, MPICH-G2, MPI_Connect1, PACX-MPI, MagPI 2 and Stampi 3 have been studied for connecting different MPI implementations. Conventional parallel programming is implemented by these MPI-like systems in tightly coupled high performance parallel computing networks or network of workstations. MPI needs middleware to interact with the global grid.

The other programming paradigm of importance is RPC (Remote Procedure Call) 2. It is used for calling remote functions through a simple programming interface. To a user, RPC presents a transparent interface to a remote function. $\mathrm{RPC}$ is the most promising candidate for Grid programming interface due to its simplicity and user friendliness. With this interface, the grid middleware can invoke a remote grid resource via RPC calls. Ninf-g [9], NetSolve-G(Grid Solve) OmniRPC [7] and so forth provide Grid RPC interface to their systems.

pyBubble 6] provides DAG(Directed Acyclic Graph)-based co-scheduling as a mechanism to minimize repeated interactions among resources. Unlike Ninf and NetSolve, our system can store the applications task dependencies in DAGs. These DAGs allow pyBubble to schedule communications with a functionalitybased scheduling algorithm. Our DAG-based Grid Runtime with RPC programming interface exhibits substantial performance improvements in terms of the execution time of related RPC requests. This efficiency increases in larger distributed systems that suffer from large data transfer overheads.

\section{Motivation}

In the traditional RPC paradigm, individual RPCs are processed independently. The actual scheduling of the remote invocation is unilaterally determined by the remote service receiving the RPC request. Clients, however, may have to meet scheduling constraints. If a remote call entails submitting a batch job or a sequence of related tasks, the client may at least want to know what the queue

\footnotetext{
${ }^{1}$ MPI_Connect, http://icl.cs.utk.edu/projects/mpi-connect

${ }^{2}$ MagPIe, http://www.cs.vu.nl/albatros

${ }^{3}$ Stampi, http://ssp.koma.jaeri.go.jp/en/index.html

${ }^{4}$ GridSolve, http://www.nsf-middleware.org/documentation/NMI-R4/0/gridsolve
} 
length is, or have some notion of the expected time of completion. Clients may also need to co-schedule multiple RPCs if the input and output parameters of these multiple RPCs are interrelated. Co-scheduling will help avoid the transmission of redundant data, resulting in an overall shortened response time and reduced network congestion. The total processing time can also be shortened by executing modules concurrently whenever possible. However, GridRPC systems do not support any mechanism to co-schedule GridRPC that targets heterogeneous and loosely-coupled systems over wide-area networks. This work is an attempt to fill this gap.

Figure1 1] illustrates two kinds of data flow involving multiple RPCs. The client invokes the services of three servers for processing a job consisting of three tasks. There is ample opportunity to reduce redundant network traffic between clients and servers when we execute this series of related RPCs. In the left diagram, servers always return the result to the client after the execution of their tasks. But, in the co-scheduled system, servers don't need to return the intermediate results to the client, but instead send the intermediate results to other servers directly as their inputs. In the right diagram, server3 executes the final task, and sends only the final result back to the client.
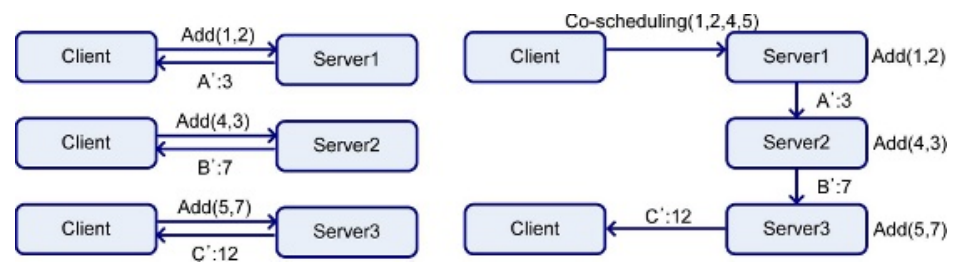

Fig. 1. Data flow compared with co-scheduling

Grid Computing usually involves the processing of very large amounts of data using distributed resources over wide area. When we take the overhead of the data transfer time into consideration, decongestion of network traffic by coscheduling in the Grid can substantially contribute toward reducing the overall response time of multiple RPCs.

\section{Framework: The pyBubble}

Our system, pyBubble, is a web service-based Grid middleware for parallel and distributed computation. This system intends to be a GridRPC system that uses XML-based RPC for the interactions between the client application and remote computing resources. pyBubble is written in the Python programming language to support portability across multiple platforms. pyBubble uses SOAP as the transport encoding and supports DAG based co-scheduling and a restart protocol for improving the dependability of the system. 


\subsection{SOAP-based Implementation}

pyBubble $[\underline{6}$ uses SOAP for performing remote procedure calls. SOAP provides an envelope that encapsulates XML data for transfer through the Web infrastructure with a convention for Remote Procedure Call (RPCs) and a serialization mechanism based on XML Schema data type. We note that other RPC mechanisms for Grids are possible, including XML-RPC5 which also uses XML over HTTP. While XML provides tremendous flexibility, it currently has poor support for binary data due to a significant encoding cost 4 . Therefore, we compress the $\mathrm{xml}$ documents before they are transferred in order to reduce the overhead caused by the substantial encoding overhead of XML.

\section{2 pyBubble Architecture and General Scenario}

Figure 2 shows each of these components of pyBubble and illustrates the relationship and data flow between the components. pyBubble consists of the client, the resource broker, and resource servers. We can get metadata of available servers by intergrating monitoring services, but we can use specific host configuration information collected from servers in this work.

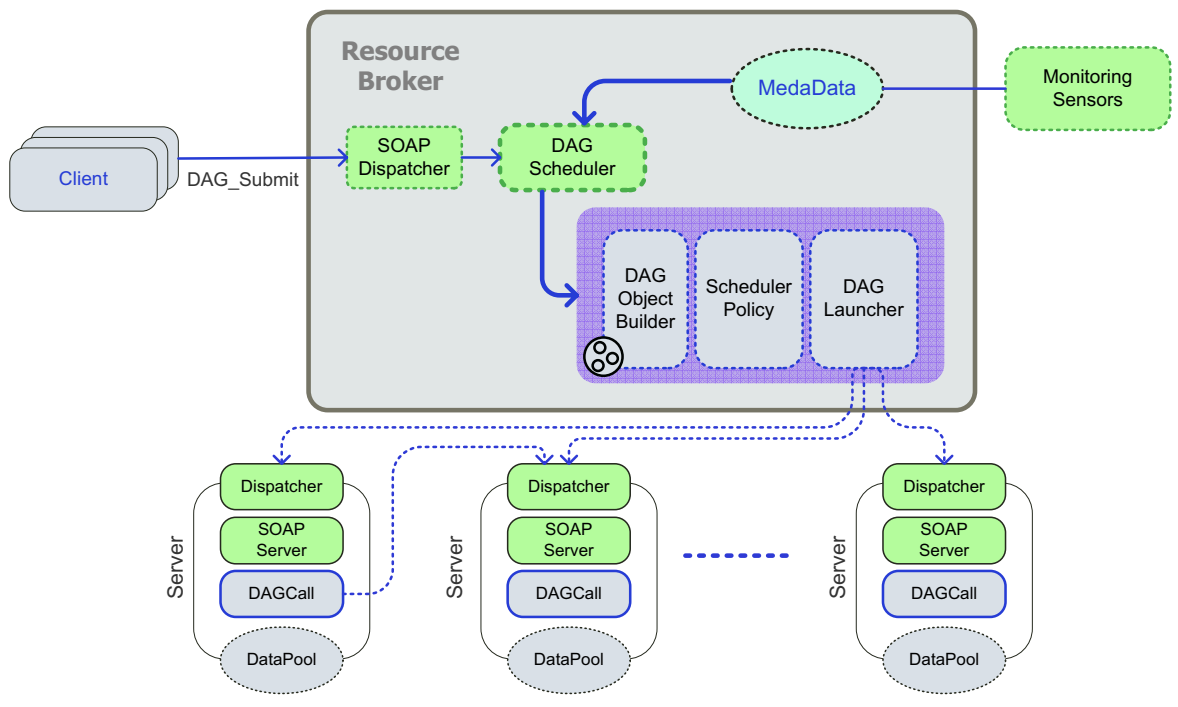

Fig. 2. The architecture of pyBubble consisting of client, broker and servers

We can assume that the user has several tasks targeted to distributed servers. First, the user sends the tasks to the pyBubble broker with the programming interface for DAG-based co-scheduling which constructs the DAG from client's tasks, subject to constraints. The client specifies the input data, the result labels and the function names with the programming interface. The tasks to be sent to

\footnotetext{
$\overline{5}$ XML-RPC, http://www.xml-rpc.com
} 
the broker should be a sequence of interrelated requests. The broker ranalyzes the relationships of input and output data within tasks, and then checks the precedence or dependency between tasks. It then constructs the DAG. The DAG has the information about intra-task relationships.

Tasks are assigned to the right server based on the scheduling algorithm because the broker has the information about available servers. To execute the tasks, first, the broker submits root tasks in the DAG. The root tasks are assigned to multiple servers to be executed in parallel. They call the respective child tasks in sequence according to the DAG. They also send the result, the scheduling information for the child task, and the DAG to the child tasks. After all the tasks are completed, the server executing the final task sends its result to the broker.

\section{DAG-Based Co-scheduling of RPC}

\subsection{The DAG Model and Application Encoding Using DAG}

The DAG is a generic model of a parallel program consisting of a set of interdependent processes (nodes). A node in the DAG represents a task. The graph also has directed edges representing a partial order among the tasks. The partial order creates a precedence-constrained directed acyclic graph[20]. For scheduling purposes, tasks are described by a DAG. A DAG incorporates all the precedence relationships among tasks and information about the assigned task in order to make scheduling decisions. After scheduling, the DAG includes information about which task is assigned to which server.

\subsection{DAG-Based Co-scheduling Algorithm with Function Availability}

After constructing the DAG, the broker schedules the DAG tasks based on function availability since the broker can get information of configuration files specified on each server and knows which resource can execute which function. Figure 3 shows the pseudocode of DAG-based co-scheduling by functional availability. The function definition procedure assists in finding the available resources offering the requested function and this returns the candidate set of the resources. The variable DAG is the collection of the requests described in DAG, and Rmax is the number of resources. ResourcePool includes the information about available resource to which the broker assigns some task. Each task in the DAG finds the cadidate resources set offering the function called by the method FindResHavingFunc. In the candidate resource set, one resource is selected for assignment to the task. After finding the root tasks, they are executed with the Execute method. 


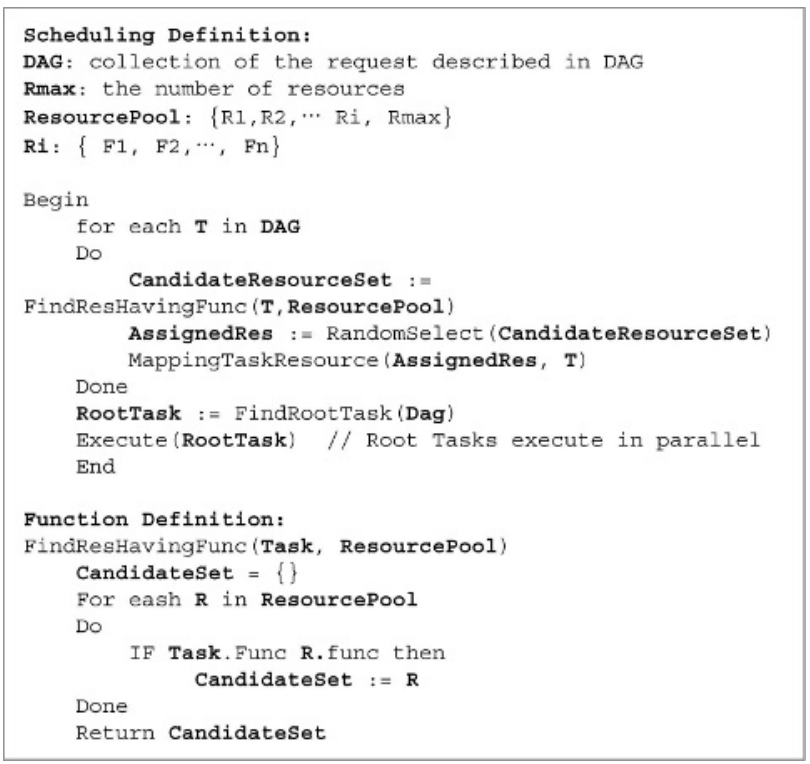

Fig. 3. Pseudocode of scheduling by functional availability

\section{Experiment Results}

The experiments compare co-scheduled RPC with conventional RPC. The performance criteria are data size, number of processors, and the CPU power. The efficiency of DAG-based co-scheduling can vary with these factors.

\subsection{Application: Image Processing}

Image Processing is appropriate for studying the efficiency of co-scheduling. In this experiment, images can be very large, on the order of several gigabytes in size. The execution of a series of transformations - in a specific order - on an image is common in image processing. This experiment shows the improved efficiency of DAG-based co-scheduling. The results of experiments depend of the combination of the function set and the DAG construction.

Comparison with increasing image size. Figure 4 shows that the execution time of conventional RPC increases sharply when the data size increases due to network traffic overhead. But the increase in the execution time of co-scheduled $\mathrm{RPC}$ is not as drastic. Parallel processing of concurrent tasks and the resulting reduction in redundant network traffic contribute to this improved performance.

Comparison on the basics of CPU Performance. This experiment uses $\mathrm{CPU}$ power as the performance criterion: the high performance group and the low 


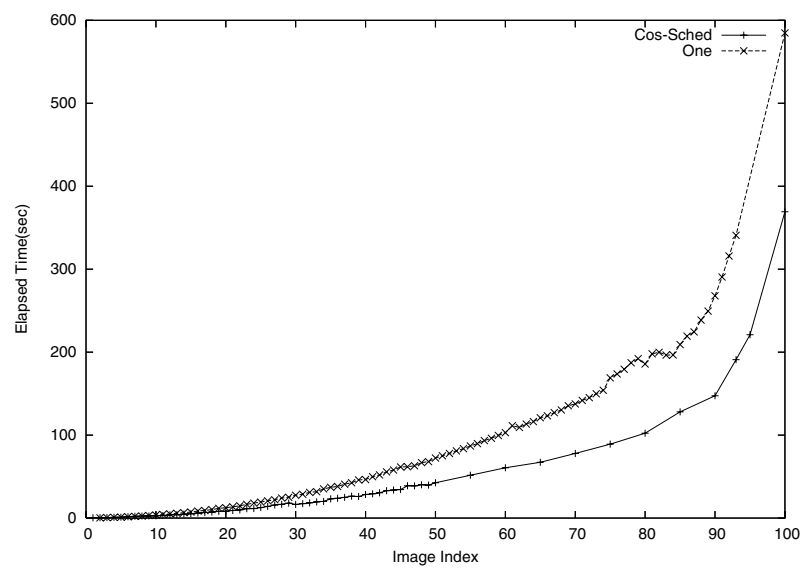

Fig. 4. Execution time of Co-scheduled RPC and Conventional RPC

performance group. Figure 5 compares co-scheduled RPC with conventional RPC in both the server groups. the performance of the low performance group is not improved significantly with co-scheduled RPC because that has large overhead for co-scheduling tasks and is not less afftected by reducing network overhead.

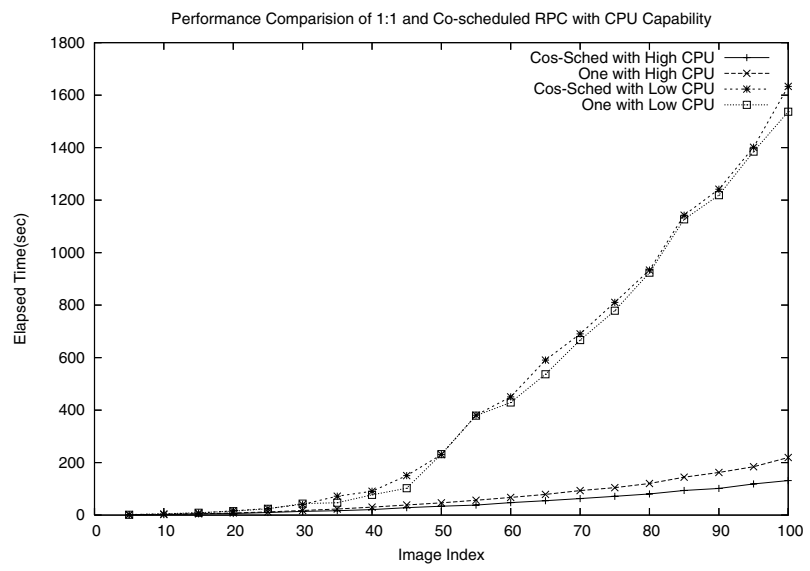

Fig. 5. Comparison of conventional and co-scheduled RPC in low performance CPU group and high performance RPC group

Comparison with the different number of processors. In figure 6, the single processor records the worst performance as expected. Two and three processors achieve the best performance. The DAG has three root tasks and two child tasks and hence two or three are just the right numbers. Two processors return a performance below that of three processors. This is understandable in light of the fact that there are three (concurrent) root tasks in the DAG. Four and five processors also exhibit good performance until the number of images reaches 80 . Thereafter, the performance degrades due to heavy network traffic induced by large sized images. 


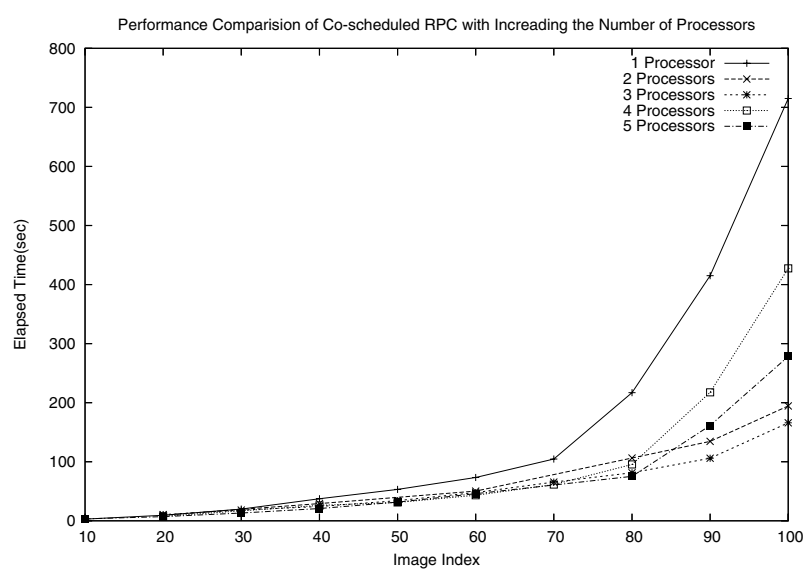

Fig. 6. Performance comparison of co-scheduled RPC with increasing of processor

\section{Conclusion}

In this paper we have proposed a DAG based co-scheduling technique as a tool for efficient RPC programming in the Grid. Co-scheduling intends to avoid redundant transmission of inputs and outputs in order to reduce network traffic congestion. The system supports a portable programming interface for DAG based co-scheduling as a user facility. DAG-based applications are scheduled using functionality- and input output data location-based co-scheduling algorithm. Image processing applications were used for test purposes and have proved that DAG based co-scheduling exhibits considerable performance improvements over conventional RPC.

\section{References}

1. Dorian C. Arnold, Dieter Bachmann, and Jack Dongarra. Request sequencing: Optimizing communication for the grid. In Euro-Par, pages 1213-1222, 2000.

2. Gregory L. Field Lola Gunter Thomas Klejna Shankar Lakshman Alexia Prendergast Mark C. Reynolds David Gunter, Steven Burnett and Marcia E. Roland. Client/Server Programming With Rpc and Dce. Que, 1995.

3. GlobalGridForum. http://www.ggf.org.

4. M. Govindaraju, A. Slomenski, V. Choppella, R. Bramley, and D. Gannon. Requirements for and evaluation of rmi protocols for on the performance of remote method invocation for scientific computing. In Proc. of the IEEE/ACM International Conference on Supercomputing (SC 2000), November 2000.

5. Ian Foster, Carl Kesselman, editor. The GRID2: Blueprint for New Computing InfrastructureMeyers. Morgan Kaufmann, 2003.

6. DongWoo Lee and JiHyun Choi. http://pybubble.sourceforge.net, 2004.

7. Mitsuhisa Sato, Taisuke Boku, and Daisuke Takahashi. Omnirpc: a grid rpc ystem for parallel programming in cluster and grid environment. In CCGRID, pages 206-, 2003. 
8. Keith Seymour, Hidemoto Nakada, Satoshi Matsuoka, Jack Dongarra, Craig A. Lee, and Henri Casanova. Overview of gridrpc: A remote procedure call api for grid computing. In GRID, pages 274-278, 2002.

9. Y. Tanaka, Hidemoto Nakada, Satoshi Sekiguchi, Toyotaro Suzumura, and Satoshi Matsuoka. Ninf-g: A reference implementation of rpc-based programming middleware for grid computing. J. Grid Comput., 1(1):41-51, 2003. 